\title{
La remonte d'une unité méhariste: son caractère polymorphe. Conditions d'une homogénéité moyenne
}

\author{
par le Vétérinaire Capitaine $A$. BOUÉ
}

\section{L'ASPECT POLYMORPHE D'UNE UNITÉ MÉHARISTE}

UNE unite méhariste ne présente pas toujours le lot d'animaux adéquats, capables de réaliser le travail imparti. S'y côtoient souvent des méhara de qualité fort inégale; près de certains, excellents à subir les fatıgues des missions lointaines ou urgentes, d'autres, défectueux ou tarés, n'offrent que le seul avantage de réaliser le quantum des effectifs. La résultante d'un tel état de fait, sans vouloir en considérer le côté pécuniaire, est une baisse indéniable du potentiel militaire.

Cette diversité, bien qu'existant dans bon nombre de détachements, ne se présente pas fort heureusemént avec la même amplitude ; elle est relativement plus grande dans la petite unité (douair, maghzen, goum) que dans une grande unité telle que la compagnie saharienne; elle apparait donc dans sa relativité inversement proportionnelle au nombre d'animaux.

Le dimorphisme s'accuse par différentes modalités. Généralement, il s'agit d'animaux dont la morphologie ne correspond pas au dur service envisagé. De tels sujets manquent d'ossature, de charpente; sans ampleur, étriqués, ils apparaissent serrés dans leurs deux bouts; leurs membres grêles supportent un corps étroit. La masse qui conditionne la solidité est absente. Le modèle est trop léger car insuffisamment établi. Souvent, le lymphatisme vient accentuer ce défaut, conférant au sujet le vocable " commun ». Sans énergie. manquant de " sang », l'animal ne possede pas la trempe qui est le facteur $\mathrm{du}$ fond. Le rendement obtenu ne sera pas celui escompte.

Habituellement, le dromadaire morphologiquement insuffisant est affligé d'une petite taille; l'amplitude du pas est fonction de la hauteur. Des tailles très différentes révèlent au premier coup d'œil la fantaisie qui a pu se glisser dans le choix de la remonte. C'est ainsi qu'il nous a été permis de rencontrer près d'animaux mesurant $\mathrm{l} \mathrm{m} .81$ et $\mathrm{l} \mathrm{m} .83$, certains de $1 \mathrm{~m} .71,1 \mathrm{~m} .69$ et $1 \mathrm{~m}$. 65 .

L'animal entier vient apporter la confusion dans une unité ; irritable, peu maniable, méchant à l'occasion, il est quasi inutilisable pendant la période du rut (de novembre à mars). On le tient à l'écart, on ne peut le laisser approcher des caravanes ou des campements nomades ou il sèmerait la panique.

Parfois, on rencontre des animaux trop jeunes (2-3 ans) ou trop vieux (17-18 ans). Ils apportent avec eux tous les inconvénients inhérents à leur âge. Le jeune ne peut offrir à un travail trop précoce qu'un organisme bien peu résistant car non encore épanoui; quant au vieux, il supporte le poids des ans, et son corps affaibli, plus ou moins usé, voire ruiné, ne répond plus avec la même aisance aux injonctions de son propriétaire.

L'usage peut révéler chez quelques-uns l'absence de tout débourrage et même une certaine rétivité. L'animal ne peut être sellé que dans certaines conditions bien définies : le soir ou pendant la rumination par exemple. Il en est même dont on ne peut se saisir que par la force ou par la ruse.

Enfin, plus rarement, on trouve desi méhara tarés, leur nombre vient néanmoins grossir celui des indésirables et augmenter l'hétérogénéité de la remonte.

$\mathrm{Ce}$ caractère polymorphe peut venir :

- d une indifférence coupable dans le choix des animaux. On est alors bien peu exigeant et l'on se contente de ce qui s'offre. Cette indifférence confine à la négligence;

- d'une connaissance empirique accordant trop d'importance à des signes secondaires sans valeur sans envisager d'autres facteurs plus importants;

- d'une certaine ignorance en la matière. On prête dès lors au méhariste une confiance qui apparâ̂t toujours exagérée car les qualités de sa monture se révèlent plus subjectives chez lui que réelles chez elle;

- de grosses difficultćs dans la prospection d'animaux dues à un cheptel local peu important ou déficient par la pauvreté des pâturages ou l'incurie des éleveurs : ce qui ne permet pas toujours de satisfaire correctement les besoins.

L'incidence de ce polymorphisme est toujours préjudiciable car ces dromadaires inaptes sont 


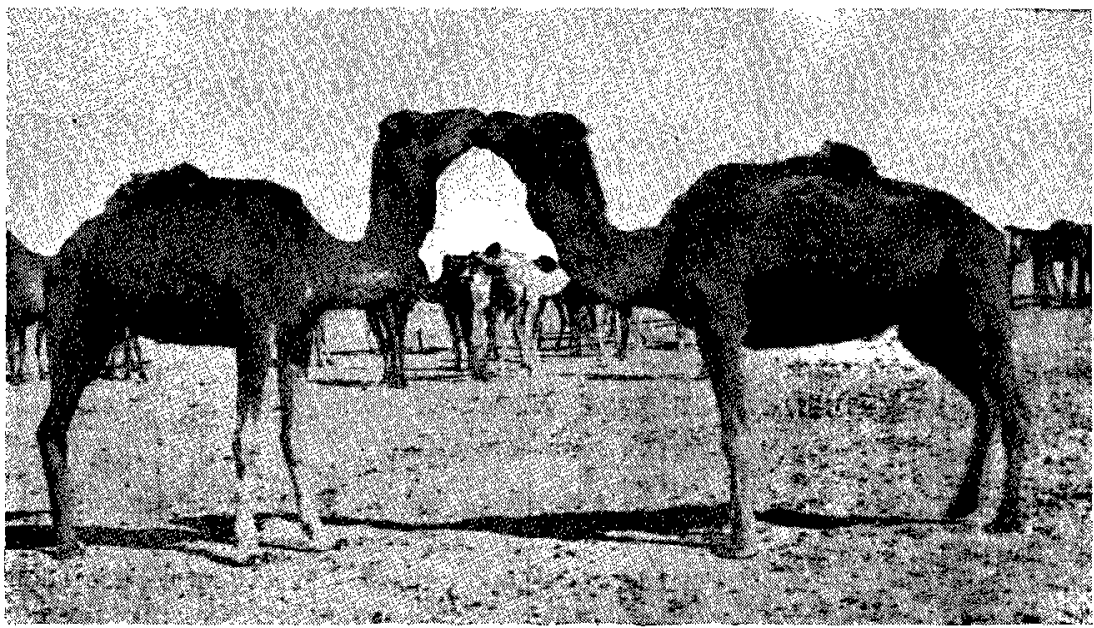

Méhara d'un modele trop léger.

poitrine large et profonde, large afin qu' « éclaté », ce soit un bon porteur. Une poitrine haute et profonde sera un indice précieux en faveur de la résistance et du fond.

Le méhari de cadre sera un animal de race, un pur sang. Il devra accuser du "sang 》 dans une élégante silhouette; sous une peau fine se détacherontmuscles et vaisseaux; le regard sera vif, l'oreille mobile par un influx nerveux sensible. II

laissés la majeure partie de l'année, pour ne pas dire l'année entière, au pâturage; ils sont une servitude sans profit. Si on les' utilise, ils alourdissent le détachement, ralentissent l'allure du peloton ou ne rejoignent qu'à l'étape. Ils déséquilibrent l'unité dans ses possibilités tactiques. Fatigués, surmenés avant de tomber épuisés et de devenir cadavres, ils jalonnent les itinéraires. « Mort en mission » : telle est la formule consacrée du compte rendu, Aussi, les quelques principes directeurs suivants pourront servir de base à l'achat ou à l'acceptation d'un méhari; ils élimineront d'éventuelles surprises qu'un examen superficiel risquerait de réserver uitérieurement.

\section{CONDITIONS D'UNE REMONTE HOMOGÈNE}

Nous envisagerons successivement les conditions présidant au choix d'une monture et comme corollaire les divers motifs de réforme.

\section{I. - Conditions nécessaires présidant au choix d'un méhari.}

L'achat d'un animal s'il est destiné à la « Remonte Etat 》 ou son acceptation s'il est présenté par un saharien est subordonné à l'examen des divers points suivants : le modèle, le sexe, l'âge, la taille, les défectuosités et les tares, l'embonpoint, l'état de santé, le dressage et l'acclimatement. L'animal doit être examiné «nu », sans harnachement; il sera sellé pour l'épreuve du dressage.

Le modèle. - Le méhari doit être jugé en fonction de son emploi : monture de gradé ou de rang ou encore est-il prévu pour la masse de transport. Dans tous les cas, l'animal devra présenter une aura de la noblesse dans le port de la tête et dans ses attitudes; le «sang» fait présumer d'une grande endurance car il augmente les qualités intrinsèques du sujet. Son élégance et sa distinction lui seront données par une encolure et des membres longs et par une relative finesse dans son squelette; si cette finesse dans l'ossature est acquise au détriment de sa solidité, une monture de cadre n'est généralement que peu chargée. Un bon méhari Targui (Touage, Tighaghaert, Tobol) ou Reguibi fera une excellente monture de gradé.

Le méhari de troupe sera bien charpenté avec de la trempe, il devra posséder du « sang sous la masse ». Rappelant l'animal de bât par un corps ramassé, un squolette fort, des masses musculaires bien développées, une encolure fournie portant une tête assez forte, il tiendra de l'animal de selle par la' longueur de ses rayons, de ses muscles, par son énergie et par son « sang » qui lui donneront une belle prestance alliée au fond. Les méhara Chamba pour leurs multiples qualités sont très appréciés dans le rang tant au Sahara Oriental que dans I'Occidental; leur réputation va de Gabès à l'Océan. Notons également les Ouled Sidi Gheikh, les Saharaoua et les métis dus à des croisements heureux Touareg, Reguibat avec Chamba; il est aussi des Reguibat et des Touareg qui, suffisamment étoffés, trouvent leur place dans le rang.

La "masse de transport 》 est constituée par les animaux de bât servant à transporter les impedimenta d'un peloton dans une compagnie saharienne. Ils doivent donc présenter une solide constitution avec un minimum de trempe: ce sont des méhara de troupe où le « gros » prédomine sur' le "sang» mais ce qui n'exclut pas la qualité car ils doivent garder la même alluré que le peloton. Le type de ces animaux se rapproche de celui du 
méhari de rang mais il est moins haut et a moins de distinction; il est toutefois supérieur à celui du chameau de bât commun.

Le sexe. - Le méhari destiné à une unité doit être hongre; ceci ne souffre aucune exception. Les testicules placés sous le périnée sont facilement visibles chez l'entier. L'animal castré présente souvent près de la pointe des fesses des marques dues à des feux mis par le hongreur. Des propriétaires peu scrupuleux marquent ainsi leurs chameaux cryptorchides afin de laisser supposer l'émasculation.

Accepter un entier pour le faire castrer par la suite est un mauvais calcul car, si l'opération ne comporte généralement que peu de risques, leur possibilité n'en demeure pas moins, et le procédé indigène, par la forte hémorragie qu'il provoque, exige une indisponibilité de deux mois environ.

L'âge. - L'âge peut être connu relativement facilement par l'examen des incisives et des canines du maxillaire inférieur.

- A 4 ans, le chameau a encore ses dents de lait qui sont d'ailleurs très usées. Ces dents caduques sont petites, peu volumineuses.

- A 4 ans et 6 mois, les deux pinces de lait tombent, les définitives apparaissent larges et plates.

- A 5 ans, ces nouvelles pinces sont complète. ment sorties, elles sont en contact avec le bourrelet supérieur, elles sont dites « à la table».

- A 5 ans et 6 mois, les mitoyennes de lait tombent. les remplaçantes sortent.

- A 6 ans, les mitoyennes sont à la table.

- $\AA 6$ ans et 6 mois, les coins de lait tombent, les remplaçants apparaissent.

- A 7 ans, les coins de remplacement sont à la table; les canines de lait peuvent être tombées.

- A 8 ans, les canines définitives sont sorties de moitié: le bord des pinces est légèrement entamé par l'usure.

- 9 ans, les canines ont atteint leur volume définitif; chez le hongre, la canine est moins

développée que chez l'entier. La surface d'usure ou " taible» des pinces est ovale.

- F. 10 ans, la table des mitoyennes est ovale.

- A 11 ans, les caractères de la table deviennent moins precis, celle des pinces toutefois s'arrondit; lovale de la table des coins est en général mal dessiné. Parfois, il peut se trouver que la table des pinces soit ronde mais les mitoyennes et les coins sont alors peu usés.

Dans certains pâturages où par exemple le tahla (Acacia tortilis) prédomine, l'usure des dents est acceléree.

L'âge minimum exigible est 6 ans, l'animal est " fait ». Dans une Compagnie saharienne, on peuf prendre des méhara à 5 ans, l'animal passant sa sixième année au détachement des deuxièmes montures. Il ne faut pas accepter d'animaux au-delà de 9 ans.

La taille. - Elle permet de distinguer le " méhari » du « djemel». Chez le méhari de cadre, on doit exiger un minimum de $1 \mathrm{~m}$. 90 au garrot, I m. 85 pour le méhari de troupe et I m. 80 pour I'animal de bât.

Dans certaines régions montagneuses, difficiles, où les animaux peuvent manquer de taille, dans le Tassili des Ajjer par exemple, il peut être bon d'accepter des animaux de la région. Il faut alors considérer l'importance des missions locales ou lointaines et discerner l'utilité d'une remonte partielle ou totale en animaux de la région. Pour les missions lointaines, on a intérêt à prendre des chameaux de format important ayant de la taille. 
La hauteur d'un chaneau peut se mesurer avec une toise même de fortune. Les membres étant à l'aplomb, la toise est placée à égale distance entre la pointe du coude et la partie antérieure de l'avantbras, le bras mobile de la toise vient s'appuyer sur le garrot; cette dimension n'est pas sujette à variation comme celle prise à la bosse. Évidemment, nous ne prétendons pas qu'un chef de peloton doive emporter dans son « guesh », une toise en' vue d'un achat éventuel; il lui sera facile d'apprécier la hauteur par comparaison avec celle connue de trois ou quatre chameaux de son unité. On procédera de la même façon avec un animal peureux.

Défectuosités et tares. - En tournant autour de l'animal, en l'examinant dans ses parties, on recherchera les défectuosités et les tares, on en remarquera les beautés également.

- La tête du méhari est portée haut, au-dessus de la ligne du dos.

- Les narines doivent être nettes; la droite porte la "rzéma ". La déchirure de la narine peut être la conséquence d'un accident ou le témoignage de l'indocilité du sujet. Ceci tare l'animal, le déprécie et attire l'attention sur son dressage. Deux narines déchirées le font refuser même pour le bât.

- Les aplombs doivent être normaux; habituellement, le chameau est légèrement panard du pied, mais si la panardise affecte les rayons, supérieurs, les coudes se rapprochent du corps et leurs callosités pourront frotter sur le thorax, les poils y seront alors usés. Cette mauvaise conformation peut donner par la suite I'hypertrophie des callosités du coude ef du sternum ( 1 ' " elbow-brushing 》 des Anglais). Un tel animal est à rejeter.

- Les articulations et les tendons doivent être nets, sans engorgement ni déformation.

- Il faut rechercher un bon dessus; une bosse située en arrière sera moins fréquemment blessée et la selle sera mieux « assise ».

- La cuisse doit être bien remplie et bien descendue.

- On doit s'assurer que le tendon d'Achille ne soit pas luxé. Quand l'animal baraque, le tendon glisse latéralement; il éprouve une certaine difficulté quand il se lève.

- Le piédestal (ou kerkera) bien dessinć doit présenté un profil net.

- La déformation congénitale du cou due au ligament de la nuque anormalement court est rare; il y a convexite anormale des premières vertèbres sans déformation latérale. Animal à refuser.

L'embonpoint. - On le juge surtout par l'état de la bosse. Il est indispensable de toucher cette bosse pour en découvrir le volume sous les poils qui peuvent y être très longs et donner ainsi l'iliusion d'une bosse replète. Une bosse bien dodue signe un bon état de santé et permet d'éliminer, en principe, la suspicion d'une maladie chronique. En effet, si le mauvais état d'entretien n'est pas dû à un travail forcé ou à un pâturage insuffisant, il est la résultante d'un trouble morbide.

Si un chameau maigre est présenté, 'on peut l'accepter s'il est nécessaire d'exercer sur les poils de la queue une forte traction pour les arracher; il faut le refuser si les poils s'arrachent facilement car, dans ce cas, son mauvais état ira s'accentuant.

Certains nomades pratiquent l'ablation' de la bosse, une large cicatrice est cachée sous les poils; le chameau ainsi diminué est à refiser.

L'état sanitaire. - Un animal en bonne santé a le poil luisant, il fouaille de la queue.

- Les dépilations font suspecter la gale. Elle apparait d'abord aux flancs, à l'aine, sur la croupe, à la base de la queue et aux,ars, puis, plus tard, à la tête, au cou, à l'abdomen, sur les lombes, le thorax et les membres. Quelquefois, la tête est la région la première atteinte; la bosse est, généralement, contaminée la dernière. L'animal tente de se gratter, de se mordre les flancs et l'ars pour calmer le prurit. Il faut être très circonspect quand l'animal a eu une application de goudron même à titre préventif car si ce n'est pas un galeux, c'est peut-être un contaminé.

- La rumination est un signe sémiologique favorable.

- Une respiration accélérée, si elle n'est pas provoquée par une course rapide, a très souvent pour origine une douleur plutôt qu'une affection pulmonaire.

- Les excréments doivent être normaux. Il faut, néanmoins, savoir qu'un changement de nourriture et la contention d'un animal nerveux sont 'suffisants pour donner de la diarrhée. Des crottins coiffés de mucosités gluantes, des fèces dysentériques commandent le refus.

- Par la palpation, on se rendra compte de l'étot du garrot et des lombes, lieux. d'élection des blessures de harnachement. La décision ne sera prise qu'après avoir estimé la gravité de la blessure le cas échéant. Une fistule au niveau des apophyses transverses des vertèbres lombaires entraîne le refus. Une plaie à la bosse est de guérison difficile.

- L'intégrité des callosités s'observe lors du baraquer ou du lever de l'animal. Il aura quelque velléité à s'appuyer sur une callosité endommagée, il cherchera ensuite à soulager la pression qui $\mathrm{y}$ est exercée en rejetant le poids de son corps sur une autre région. L'animal baraque franchement s'il n'appréhende aucune douleur. Toutefois, le chameau 


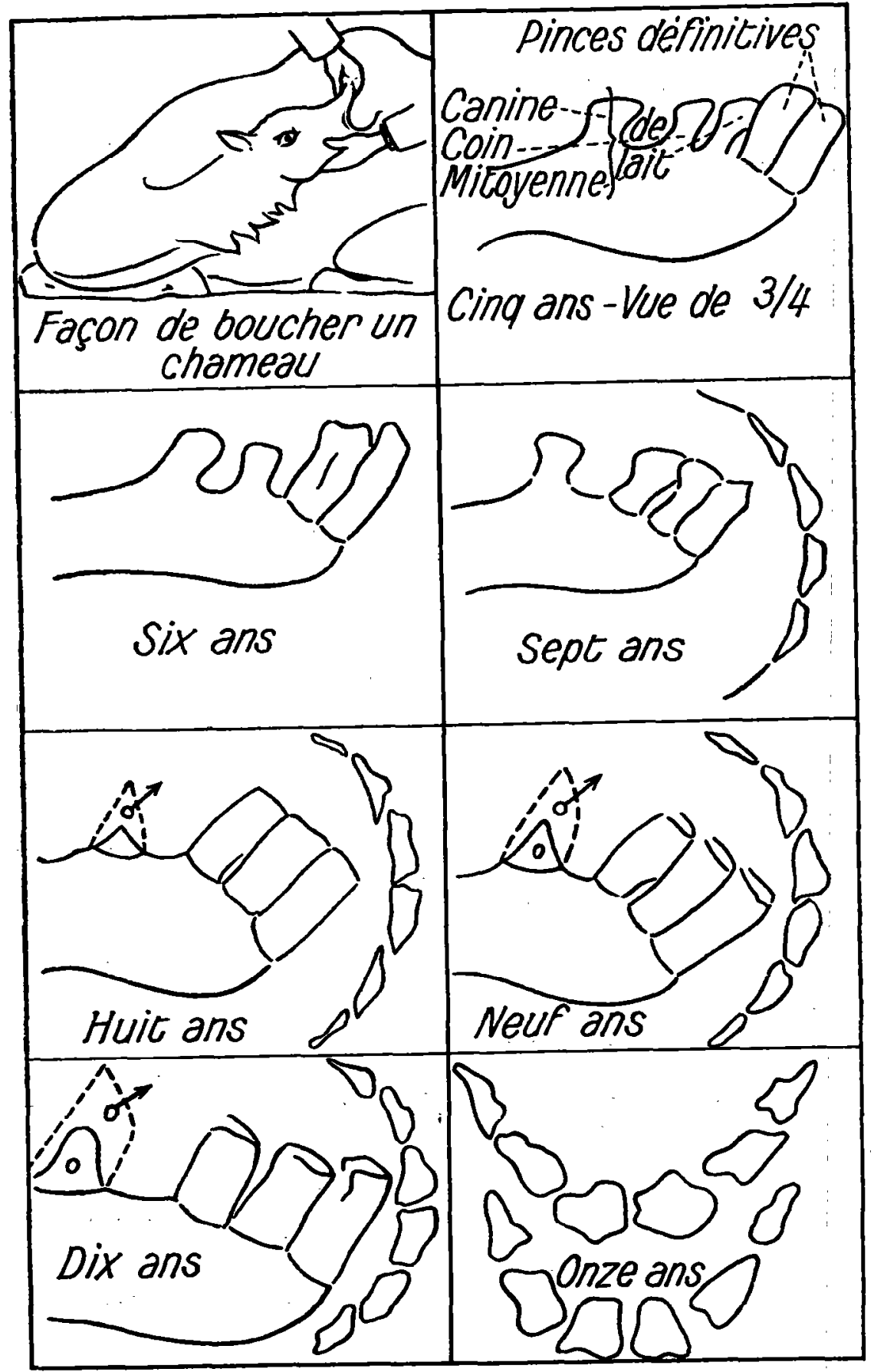


venant de l'erg, habitué au sable, marquera un temps d'hésitation si on le fait baraquer sur un sol rocheux. Une callosité douloureuse fait surseoir à l'acceptation du méhari.

- I'intégrité des soles se considère au pas et au trot. Toute sole douloureuse, crevassée, excoriée, suintante, "cuite " fait suspendre l'incorporation de l'animal. D'autre part, le trot permettra de juger de la facilité des allures, de voir si l'animal se « coupe»; dans ce cas, c'est le refus qu'il faut prononcer.

L'haleine fétide observée dans la gangrène de l'expansion palatine doit être différenciée de l'haleine mauvaise consécutive à la consommation d' " arak » (Salvadora persica).

Il n'est pas négligeable de considérer les feux. Certains sont des marques de propriété, d'autres unt élé placés dans un but thérapeutique; bien qu'il ne faille pas toujours leur accorder une grande importance, car leur logique n'est que bien rarement [ustifiée, ils permettent, néanmoins, d'attirer l'attention sur la région et de la mieux considérer. Par exemple, un feu en $\mathrm{O}$ autour de l'orbite doit faire suspecter un trouble grave de la vision. Il convient de signaler que ces divers feux avec leur place et leur forme peuvent revêtir une certaine importance pour établir le signalement.

Le dressage. - L'épreuve de dressage ou pour mieux dire de débourrage n'est pas à négliger. Le méhari sellé est monté et exercé aux changements d'allure et de direction. C'est en quelque sorte l'essai direct de l'animal.

L'acclimatement. - Ce point n'est à envisager qu'exceptionnellement quand le méhari vient d'une région lointaine, car il doit être adapté au sol et à la flore. L'adaptation du pied au terrain ne prend de l'importance que si un service immédiat est demandé, sinon en laissant l'animal au pâturage l'adaptation se fera progressivement dans un temps fonction de la différence de souplesse des terrains.

La nature d'un nouveau pâturage retentit sur un organisme non acclimaté, le sujet s'entretient mal si, toutefois, il ne disparait pas. Ceci se vérifie chez les animaux venant de certains confins soudanais où la flore ne comporte que peu ou pas de plantes salées nécessitant des cures de seli transplantés dans des régions Nord où la flore est, en partie, salée, leur acclimatement est souvent difficile.

\section{II. - Conditions suffisantes pour la réforme d'une monture.}

L'anirial qui ne peut plus rendre aucun service appréciable doit être rayé des contrôles sans sursis. Les divers motifs de réforme sont : l'usure générale, la vieillesse, les troubles de la vision, la boiterie chronique, le phlegmon sous-scapulaire, la séquelle vicieuse du piédestal, la rétivité.

L'usure générale. - Cette usure se caractérise soit par un mauvais état général permanent et définitif non lié à une question de pâturage, soit par un organisme ruiné dans l'une de ses principales fonctions, interdisant la pleine utilisation de ses moyens. Avec l'âge, cette usure s'apparente à la ruine sénile.

La vieillesse. - Tout animal au-delà de 15 ans est à éliminer; on peut surseoir jusqu'à 16 ans pour un animal en très bon état et encore vigoureux. Malgré les grandes fluctuations de la table dentaire par le pâturage à partir de la quatorzième année, on admet qu'à 15 ans les pinces sont triangulaires et qu'à 16 ans les mitoyennes le deviennent. Les incisives très usées s'espacent. Dans certaines régions, des chameaux de 12 et 13 ans peuvent marquer 16 et 11 ans par suite d'une usure excessive due aux pâturages d'arbres et d'arbustes; l'état $d$ 'entretien baisse progressivement et la mise en condition ne peut plus se faire qu'artificiellement par un régime spécial, dattes ou grains; on doit réformer l'animal. Ceci illustre bien le retentissement de la nature du pâturage chez le chameau plus que chez tout autre animal.

Les troubles de la vision. - Le chameau borgne est encore utilisable bien qu'il soit sujet aux chutes en pays accidenté.

La boiterie chronique. - Le phlegmon sousscapulaire. - Avec de l'inappétence partielle, une boiterie, une baisse d'état justifie l'élinnination.

La séquelle vicieuse du piédestal. - La rétivité - Il s'agit de la rétivité acquise par une blessure douloureuse, une cicatrisation vicieuse provoquant une certaine phobie.

Ces quelques considérations permettront de fixer des idées souvent primitives et empiriques. 